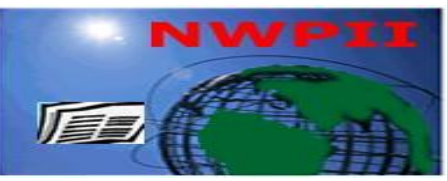

American Journal of Biomedical Sciences

ISSN: 1937-9080

nwpii.com/ajbms

\title{
Cellular Internalization and Cytotoxicity of Aptamers Selected from Lung Cancer Cell
}

\author{
Li Xu ${ }^{1}$, Zhen Zhang ${ }^{1}$, Zilong Zhao ${ }^{1,2}$, Qiaoling Liu ${ }^{1}$, Weihong $\operatorname{Tan}^{2}$, Xiaohong Fang ${ }^{1}$ *
}

\author{
${ }^{1}$ Beijing National Laboratory for Molecular Sciences , Key Laboratory of Molecular Nanostructure and \\ Nanotechnology, Institute of Chemistry, Chinese Academy of Sciences, Beijing, P. R. China \\ ${ }^{2}$ Biomedicine Engineering Center of Hunan University, Changsha, China \\ Corresponding author: \\ Xiaohong Fang, $\mathrm{PhD}$ \\ Beijing National Laboratory for Molecular Sciences \\ Key Laboratory of Molecular Nanostructure and Nanotechnology \\ Institute of Chemistry \\ Chinese Academy of Sciences \\ Beijing 100190, P. R. China \\ Email: xfang@iccas.ac.cn
}

Received:27 October 2012; | Revised:7 December 2012;| Accepted: 24 December 2012

\begin{abstract}
In this work, single-stranded DNA (ssDNA) aptamers against EGFR-transfected A549 cells, one type of non-small cell lung cancer (NSCLC) cells, were selected by cell-SELEX (systematic evolution of ligands by exponential enrichment) and were evaluated. The selected aptamers had high affinity to the A549 cells with dissociation constants in the nanomolar rang. Moreover, the aptamers were able to internalize into the cells, which is advantageous over most of the other existing aptamers. One of the selected aptamers showed significant cytotoxicity by inhibiting the cell proliferation and inducing cell apoptosis. These aptamers are expected to be new molecular probes for cancer cell targeting and drug delivery.
\end{abstract}

Keywords: aptamer, cell-SELEX, lung cancer cell, internalization, proliferation inhibition.

\section{Introduction}

Lung cancer is a leading cause of mortality in many countries [1-3]. Among the two types of lung cancer, small cell lung cancer (SCLC) and non small cell lung cancer (NSCLC), NSCLC constitutes approximately $85 \%$ of total lung cancer with high resistance to antitumor drugs and low survival rate. One of the great challenges in NSCLC treatment, as well as the therapy of other cancers, is the lack of effective cancer biomarkers and targeted drugs $[4,5]$. Several potential biomarkers have been identified, such as TP53, CEA, CA-125, and EGFR, but they still show low specificity and sensitivity in clinical practice [6]. Intensive efforts have been made to develop new molecular 
probes to selectively recognize cancer cells but not healthy cells for targeted drug delivery.

Aptamers, the short single-stranded oligonucleotides, are a new type of molecular probes selected through an in vitro method known as SELEX (systematic evolution of ligands by exponential enrichment) [7-9]. They hold a great potential in the study of molecular bases of cancer cells for cancer diagnosis and therapy. Being regarded as chemical antibodies, aptamers have the same function as antibodies in molecular recognition. With their threedimensional structures in solution and in cells, aptamers can bind specifically to their targets, ranging from small organic molecules to proteins and even whole cells. Aptamer selection by cellSELEX is suitable for the development molecular probes specifically bind to the complex surface of tumor cells with unknown molecular biomarkers. Aptamers also have many advantages over antibodies in repeatable synthesis, easy modification, long-term stability, less immunogenicity and so on. They are gaining wide applications in bioanalysis, biomedicine, and biotechnology [10-12].

Although a number of aptamers have been successfully selected against several cancerrelated proteins and some types of cancer cells [13-17], they are still at an early stage in cancer medicine. More aptamers for cancer biomarkers need to be selected and validated. On the other hand, among currently known aptamers, only a few, such as those against the prostate cancer cells and breast cancer cells, have been reported to be taken up by the cells without additional assistance [18-20]. Most aptamers cannot internalize into cells, limiting their capability as anti-cancer drug carrier for targeted therapy. In this work, we used the transfected A549 cells, one type of NSCLC cells which highly express EGFR, to select specific aptamers binding to lung cancer cells. The selected aptamers not only showed high affinity to the cells with nanomolar dissociation constants, but also were able to internalize into cells and had good stability in serum. One of the aptamers could significantly inhibit the proliferation of A549 cells after entering cells. They are promising molecular probes for cancer cell targeted therapy.

\section{Materials and methods}

\subsection{Cell culture and buffers}

Two established cell lines were used in this experiment: the non-small cancer cell A549 cells which stably transfected with EGFR-GFP (named EGFR-A549) were used as the target cells, and the untransfected A549 cells were used as control cells for counter selection. Both cell lines were kindly provided by Professor Duanqing Pei from Institute of Biomedicine and Health, Chinese Academy of Sciences (Guangzhou, China). Both cell lines were cultured in DMEM culture medium supplemented with $10 \%$ fetal bovine serum (FBS, HyClone, America), 100 units/ml penicillin-streptomycin (Sigma-Aldrich, America) and routinely grown in a humidified incubator at $37^{\circ} \mathrm{C}$ with $5 \% \mathrm{CO}_{2}$.

As for buffers, the washing buffer was prepared by adding $4.5 \mathrm{~g} / \mathrm{l}$ glucose and $5 \mathrm{mM}$ $\mathrm{MgCl}_{2}$ into 1 liter of Dulbecco's phosphatebuffered saline (Sigma-Aldrich, America). The binding buffer was prepared by adding $0.1 \mathrm{mg} / \mathrm{ml}$ yeast tRNA (Sigma-Aldrich, America) and $1 \mathrm{mg} / \mathrm{ml}$ BSA (Fisher, America) into the washing buffer.

\subsection{DNA library and primers}

The DNA library contained a central, continuous random sequence of 40 nucleotides flanked by 20 -nucleotide constant PCR primer regions (5'-ACG CTC GGA TGC CAC TAC AG (40N) CTC ATG GAC GTG CTG GTG AC-3'). The following primers annealing to the library were used for PCR during the SELEX procedure: forward primer: 5'-Cy5- ACG CTC GGA TGC CAC TAC AG; reverse primer: 5'-Biotin-GTC ACCAGC ACG TCC ATG AG. The library and primers were chemically synthesized and HPLC purified by TaKaRa (Dalian, China).

\subsection{Cell-SELEX}

The 6 nmol DNA library was unfolded in $500 \mu \mathrm{l}$ binding buffer at $95^{\circ} \mathrm{C}$ for 5 minutes and cooled on ice for 10 minutes, followed by 5 minutes incubation at room temperature. Then the ssDNA pool was incubated with EGFR-A549 cells in a $5 \mathrm{~cm}$ diameter cell culture dish (Corning, America) for 90 minutes at $37^{\circ} \mathrm{C}$ with the 
addition of $20 \%$ FBS. After incubation, cells were washed twice with the washing buffer and harvested using a cell scraper and then transferred into a $1.5 \mathrm{ml}$ tube for centrifugal washing for 3 times. After that, DNA sequences bound to target cells were separated by heating at $95^{\circ} \mathrm{C}$ for 10 minutes. The obtained DNA pool was then applied to A549 cells with the same binding condition. After incubation, the supernatant was gathered for PCR amplification.

Separated DNA pool was amplified with Cy5- and biotin-labeled primers by two-step PCR (hot start at $95^{\circ} \mathrm{C}$ for 3 minutes, 1 cycle; denaturation at $94^{\circ} \mathrm{C}$ for $30 \mathrm{sec}$, annealing at $59^{\circ} \mathrm{C}$ for $30 \mathrm{sec}$, extension at $72^{\circ} \mathrm{C}$ for $20 \mathrm{sec}, 10$ 18 cycles) [21]. In the first step PCR, $30 \mu \mathrm{DNA}$ pool was used as template and then $5 \mu$ product was used as template in the second step PCR under the same conditions. The final PCR products were separated on $4 \%$ agarose gel.

The double-stranded PCR products were separated for single strand by streptavidin-coated sepharose beads (Amersham Biosciences, America), washed with PBS and then denatured by $0.2 \mathrm{M} \mathrm{NaOH}$. The separated ssDNA solution was passed through a desalting Nap-5 column (GE Healthcare, America). The selected Cy5labeled ssDNA pool was collected and used for next-round selection and flow cytometric analysis.

To acquire aptamers with high affinity and specificity, the selection pressure was enhanced gradually by decreasing the incubation time with the target cells (from $1.5 \mathrm{~h}$ to $0.5 \mathrm{~h}$ ), increasing incubation time with the control cells (from $1 \mathrm{~h}$ to $1.5 \mathrm{~h}$ ). After 23 rounds of selection, the enriched ssDNA pool was amplified using unmodified primers and cloned into Escherichia coli using the TA cloning kit (TaKaRa, China) before sequencing.

\subsection{Flow cytometry analysis}

To monitor the enrichment of the ssDNA pool and evaluate the binding affinities of selected aptamers, flow cytometry was used after different rounds for FITC- or Cy5- labeled ssDNA. Various concentrations of the aptamers were incubated with $3 \times 10^{5}$ cells in $200 \mu \mathrm{l}$ of binding buffer with $20 \% \mathrm{FBS}$ at $37^{\circ} \mathrm{C}$ for 60 minutes. A scramble DNA sequence (Rc: 5'AATTTTTTAATTATTTATATTA-3') was used as a control. Both cells of A549 and EGFR-A549 were used. The mean florescence intensity of 10 000 cells bound by different aptamers and scramble DNA sequence were obtained. For monitoring the enrichment of the DNA pool during the SELEX, the final concentration of the FITC- or Cy5-labeled ssDNA was 500 nM. For evaluating the binding affinity, the final concentration of the FITC- or Cy5-labeled aptamers was from 0 to $200 \mathrm{nM}$. The adherent cells from the dish were detached by $0.02 \%$ EDTA. The equilibrium dissociation constant (Kd) values were determined by the ligand binding analysis according to the equation: $\mathrm{Y}$ $=\mathrm{BmaxX} /(\mathrm{Kd}+\mathrm{X})[21,22]$. All the experiments were repeated for three times.

\subsection{Aptamer based ELISA}

Biotin-labeled aptamers were coated onto the strepavidin modified plates (Thermo, America) at room temperature for 2 hours. A549 cells were added with $200 \mu \mathrm{l}$ RIPA cell lyses buffer (50 $\mathrm{mM}$ Tris , $150 \mathrm{mM} \mathrm{NaCl}, 1 \% \mathrm{NP}-40,0.5 \%$ sodium deoxycholate , $0.1 \%$ SDS, $2 \mathrm{mM}$ sodium pyrophosphate , 25mM $\beta$ glycerophosphate , $1 \mathrm{mM}$ EDTA , $1 \mathrm{mM}$ $\mathrm{Na}_{3} \mathrm{VO}_{4}$, and $0.5 \mu \mathrm{g} / \mathrm{ml}$ leupeptin), and then gathered to a $1.5 \mathrm{ml}$ tube for centrifugation at 14 $000 \mathrm{rpm}$ for $5 \mathrm{~min}$. The supernatant was collected as the whole cell extract. The plate was then washed with PBS and incubated with whole cell extract protein from A549 cells at $37{ }^{\circ} \mathrm{C}$ for 2 hours. After blocked with $2 \%$ BSA, the plates were incubated with nucleolin antibody (Santa Cruz Biotechnology, America) diluted as 1:1000 according to the instructions. After this, plates were incubated with FITC-conjugated second antibody (diluted as 1:1000, Sigma-Aldrich, America), and then HRP-conjugated anti-FITC third antibody (diluted as 1:3000, LifeHolder, America). At last, solution was added with TMB to develop color and with $\mathrm{H}_{2} \mathrm{SO}_{4}$ to stop the reaction. Absorbance of the solution at $450 \mathrm{~nm}$ 
was read with a 96-well plate reader (iMark microplate reader, Bio-RAD, USA)

\subsection{Cell viability assays}

A549 Cells were cultured in 96-well plates for 24 hours and then incubated with $10 \mathrm{uM}$ different aptamers or Rc in DMEM culture medium containing 10\% FBS for 48 hours at 37 ${ }^{\circ} \mathrm{C}$. Cytotoxicity was evaluated using a WST-8 assay with a Cell Counting Kit-8 (CCK-8; DOJINDO, Kumamoto, Japan). The absorbance value at $450 \mathrm{~nm}$ was read with a 96-well plate reader (iMark microplate reader, Bio-RAD, USA).

\subsection{Confocal microscopy imaging}

A549 cells were grown to $60 \%$ confluence in a $5 \mathrm{~cm}$ diameter dish (Corning, America). To evaluate the aptamers entering into A549 cells, the cells were incubated with $2 \mu \mathrm{M}$ FITC-labeled aptamers in DMEM with $10 \%$ FBS for 18 hours at $37^{\circ} \mathrm{C}$, followed by washing twice with the washing buffer and observation under the confocal microscopy with $488 \mathrm{~nm}$ laser excitation (FV1000-IX81, Olympus, Japan).

To evaluate the aptamer-induced apoptosis, the cells were incubated with $5 \mu \mathrm{M}$ selected aptamers in DMEM with 10\% FBS for 48 hours at $37^{\circ} \mathrm{C}$. Then, the cells were washed twice with PBS and incubated with Annexin V-FITC/PI apoptosis detection kit (KenGEN Biotech, China) according to the manufacturer. At last, cells were observed by the confocal microscope with $488 \mathrm{~nm}$ laser excitation for FITC and $559 \mathrm{~nm}$ laser excitation for PI. The fluorescence signals were collected by a 10x objective (NA 0.40, UPLASPO, Olympus).

\subsection{Western blot}

To evaluate the nucleolin expression level, EGFR-A549 and A549 cells were added with $200 \mu$ R RIPA cell lyses buffer respectively, and then collected to two $1.5 \mathrm{ml}$ tubes for centrifugation at $14000 \mathrm{rpm}$ for $5 \mathrm{~min}$. The supernatants were collected as the whole protein extracts for quantitation. The protein extracts were separated with $7.5 \%$ polyacrylamide gel. After transmembrane, the NC membranes were washed twice with TBST and incubated with $3 \%$
BSA for 1 hour at $37^{\circ} \mathrm{C}$ followed by C23 (1:200, nucleolin antibody, Abcam, USA) and secondary antibody (1:20000, Rockland, America ) incubation. Total cell lysate was quantitated with anti $\beta$-actin antibodies as an internal control. At last, the images were scanned by a two-color infrared laser imaging system (LI-COR, America).

\section{Result and discussion}

\subsection{Selection of aptamers that bind to the lung cancer cells}

Since the over-expression and activation of EGFR is closely related to the staging of NSCLC and decreased chemotherapy/radiotherapy sensitivity [25], we intended to develop molecule probes targeting to high EGFR expression lung cancer cells. Therefore, EGFR-A549 cells, the NSCLC cells which stably expressing high-level EGFR, were used as the target cells to mimic the lethal lung cancer cells under physiological conditions. Original A549 cells were used as the control in the cell-SELEX.

The cell-SELEX process was monitored by flow cytometry. After each round of selections, the binding of Cy5-labeled ssDNA pools with the cells was tested. As shown in Figure 1, with the increased numbers of selection cycles, the fluorescence intensity increased from 717.5 at cycle 1 to 1850.4 at cycle 23 for EGFR-A549 cells while the population of the ssDNA above the background fluorescence intensity increased from $50.9 \%$ at cycle 1 to $96.7 \%$ at cycle 23 (Figure 1A). This indicated the DNA sequences with higher binding affinity gradually dominated in the DNA pools during the SELEX. As no further increase of fluorescence intensity was found after cycle 23, the DNA pools at cycle 23 were cloned and sequenced.

We observed that the fluorescence intensity and population of ssDNA bound to the control A549 cells also increased with the selection cycles (Figure 1B); although the intensity increase was a little lower than the target EGFRA549 cells. This situation happened in several other reported cell-SELEX that the aptamer of the target cells can also bind to the control cells used 
in counter selection $[21,25]$. We expected that the targets of the DNA pool were expressed on the surfaces of these two cell lines, and additional expression of EGFR did not alter the target expression that much on A549.

Table 1. Sequences of Selected Aptamers for A549 cells

\begin{tabular}{ll}
\hline Aptamers & \multicolumn{2}{c}{ Sequences $^{\text {a }}$} \\
\hline R50 & TAAAGGGCGGGGGGTGGGGTGGTTGGTAGTTGTTTTTTCTGTTTC \\
R14 & ATGCGAACAGGTGGGTGGGTTGGGTGGATTGTTCGGTTTCTTGA \\
R13 & TCTCTAGTTATTGAGTTTTCTTTTATGGGTGGGTGGGGGGTTTTT \\
R9 & CGGTGGGGGTGGGTAGGTAATCGATAGAGTACGGGGGGTTGGATT \\
R6 & GGTTGGTTGG GGTTGGGTTG TTTTTGGGGT GATATGGGGG TTGGA
\end{tabular}

${ }^{a}$ the primer regions of aptamers are excluded

A.

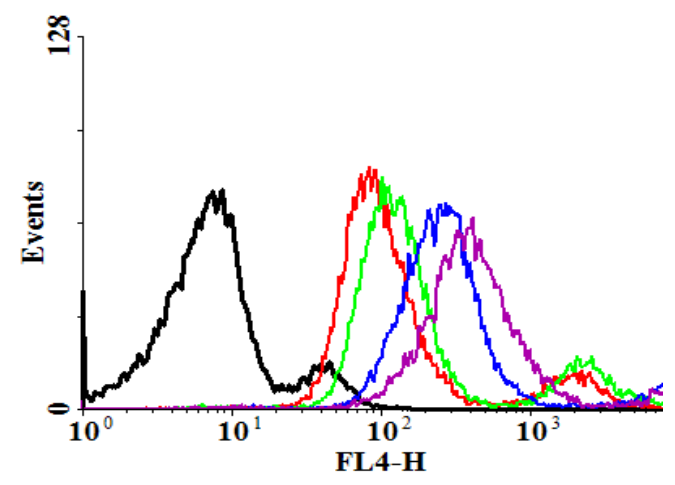

B.

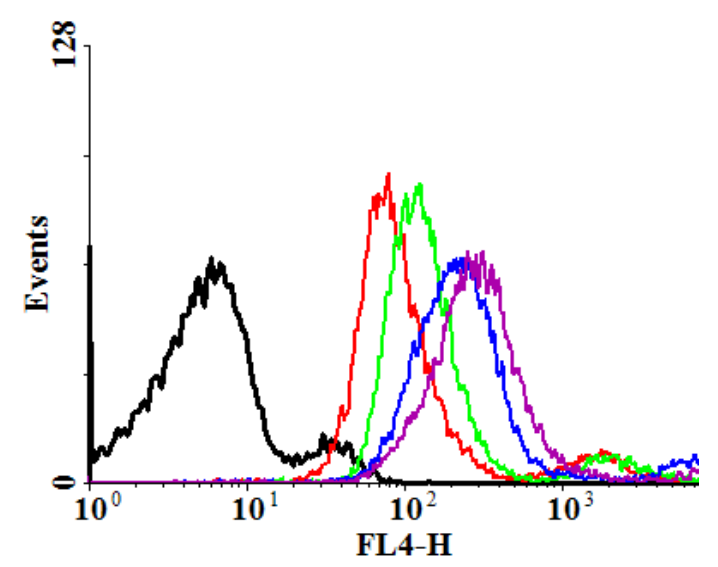

Figure 1: Flow cytometry analysis of the binding of Cy5-labeled ssDNA pools to the lung cancer cells. A: EGFR-A549, B: A549 cells. Black curve: cells without any ssDNA; red curve: ssDNA pool from the 1st cycle of selection; green curve: the 6th round ssDNA pool; blue curve: the 13th round ssDNA pool; purple curve: the 23th round ssDNA pool. Moving of the curves from left to right indicated the enrichment of ssDNA pool during the cell-SELEX.

DNA sequences from 160 clones were obtained, grouped into six families based on the homology of random sequences. Many repeats were observed in each family. The highly repeated sequences as shown in Table 1 were chosen to test respectively their ability of binding to the cells by flow cytometry.

We have further quantified the affinity of R50, R14, R13, R9, and R6 with different concentrations of the aptamers, as shown in the figure for the representative aptamer, R50 (Figure 2). The dissociation constants of R50 to A549 cells and EGFR-A549 cells were $55.4 \pm 30.6 \mathrm{nM}$ and $6.4 \pm 11 \mathrm{nM}$ respectively. Since the binding affinity of the aptamers to A549 cells was also quite high, and the EGFR-A549 cells displayed green fluorescence (EGFR was tagged with GFP) which was disadvantageous for its interference with dye-labeled aptamer imaging, we just used A549 cells as a representative lung cancer cell in our later studies. The dissociation constants of $\mathrm{R} 14, \mathrm{R} 13, \mathrm{R} 9$, and R6 to A549 cells were $29.6 \pm 11.8 \mathrm{nM}, 23.0 \pm 7.7 \mathrm{nM}, 8.9 \pm 6.5 \mathrm{nM}$, and $148.0 \pm 30.1 \mathrm{nM}$ respectively. Among them, four high affinity aptamers were studied in later experiments, including R50, R14, R13, and R9. 
A

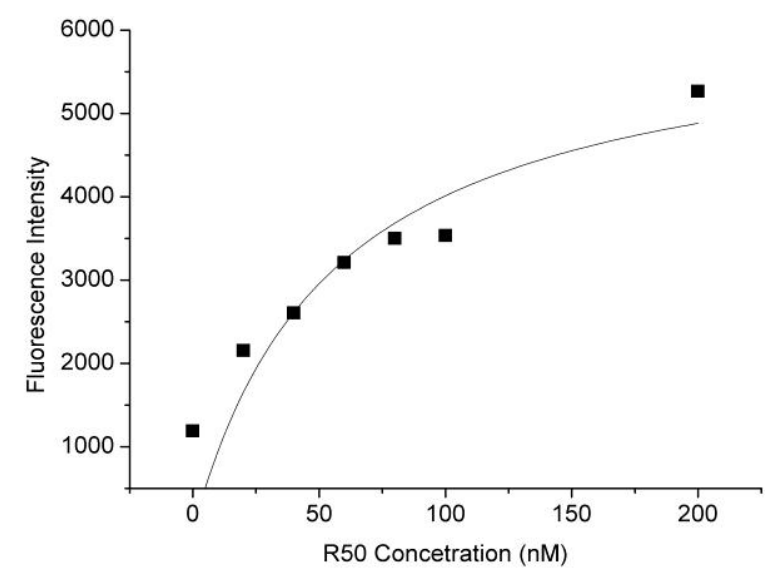

B

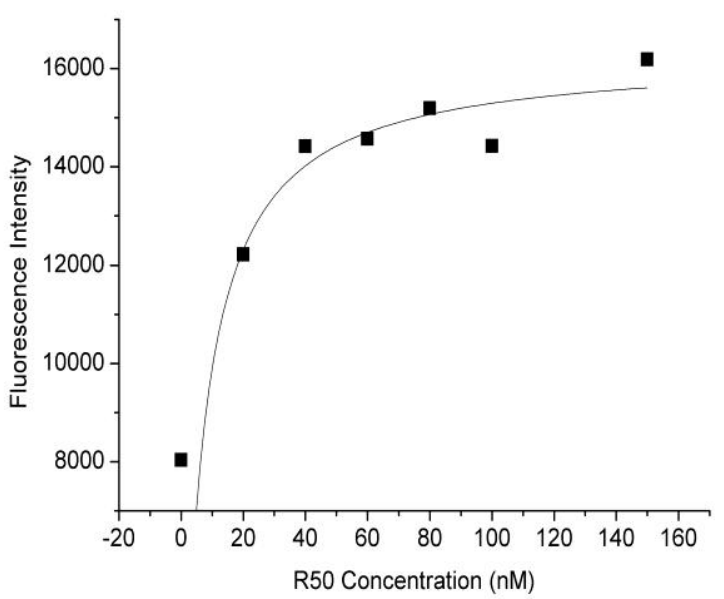

Figure 2: Affinity analysis of aptamer R50 to A549 cells. R50 was labeled with Cy5 and incubated with EGFR-A549 cells or A549 cells (Num. =106) at concentrations of $0,20,40,60,80,100$ and $200 \mathrm{nM}$. A: Binding affinity of Cy5-labeled R50 to A549 cells using flow cytometry; B: Binding affinity of Cy5labeled R50 to A549-EGFR using flow cytometry. The $\mathrm{Kd}$ value was $55.4 \pm 30.6 \mathrm{nM}$ for $\mathrm{A}$ and $6.4 \pm 11$ $\mathrm{nM}$ for B.

Although the aptamers were selected from the cells highly expressing EGFR, we found that the target of the aptamers was not EGFR, as we did not observe the colocalization of the dye labeled aptamer and GFP tagged EGFR in the confocal images of the cells. It has been reported that the target of several G-rich aptamers might be nucleolin, a type of protein highly expressed in cancer cells [24]. Since the G base content is also high in our aptamers, we performed the aptamer based ELISA with the nucleolin antibody to check whether the aptamers bind to nucleolin or not. The results showed that for the plates modified with biotin labeled aptamers of R50, $\mathrm{R} 14, \mathrm{R} 13$, and R9, more nucleolin proteins were captured, thus nucleolin antibody showed higher intensities than those with the control sequence Rc modified plate or untreated plate (Figure 3). The binding of aptamer to nucleolin but not to EGFR was also confirmed with the dot blotting experiment (Supplementary Figure S1).This indicated that the aptamers had high affinity to nucleolin. The target validation for nucleolin needs further confirmation.

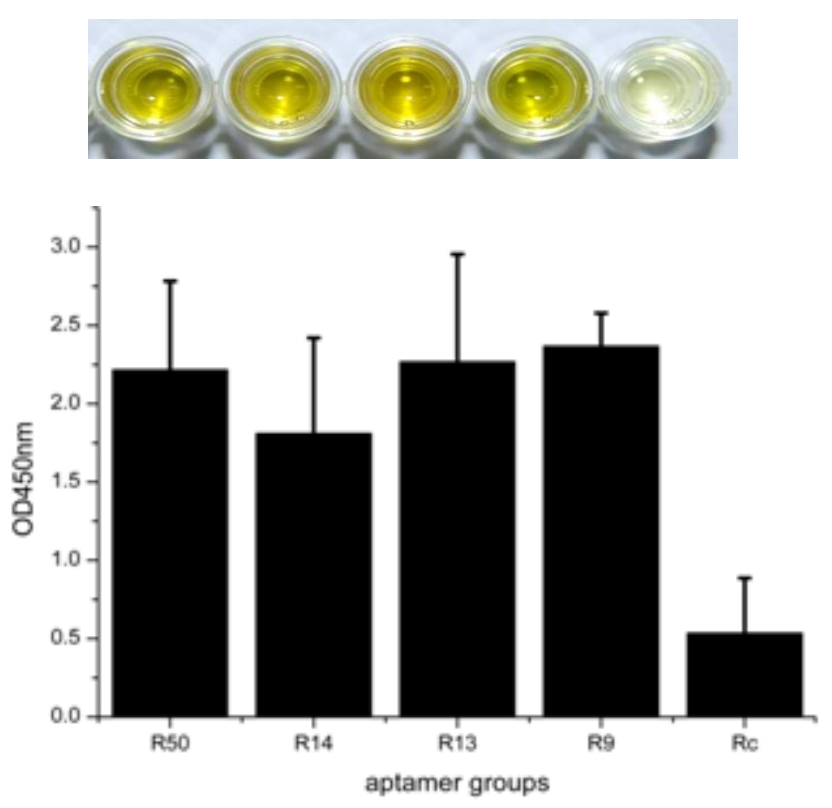

Figure 3: Binding of the biotin-labeled aptamers with nucleolin. The plate was treated with different biotin labeled aptamers R50, R14, R13, R9, and control sequence Rc. The whole protein sample from A549 cell lysates was added onto plate and then nucleolin antibody C23 was added. FITC-conjugated second antibody and HRP-conjugated anti-FITC third antibody were used to develop color. The above figure shows the color change in biotin-labeled aptamers coated plates and the figure below shows the corresponding absorbance.

We have also tested the nucleolin expression level in EGFR-A549 cells and A549 cells by immunofluorescence. As it expected, both cells expressed nucleolin, with a higher nucleolin expression in EGFR-A549 cells (Supplementary 
Figure S2). The use of EGFR-A549 cells facilitated the selection of necleolin-binding aptamers.

\subsection{Internalization of the selected aptamers into A549 cells}

A549 cells were imaged under the confocal microscope after incubation with four FITC- labeled aptamers for 18 hours. After incubation, aptamers binding to cell surface were washed off. As shown in Figure 4, all the aptamers could enter the A549 cells, and gathered around the nucleus, some of them even located into nucleus. While for the control DNA sequence, it hardly internalized into cells.

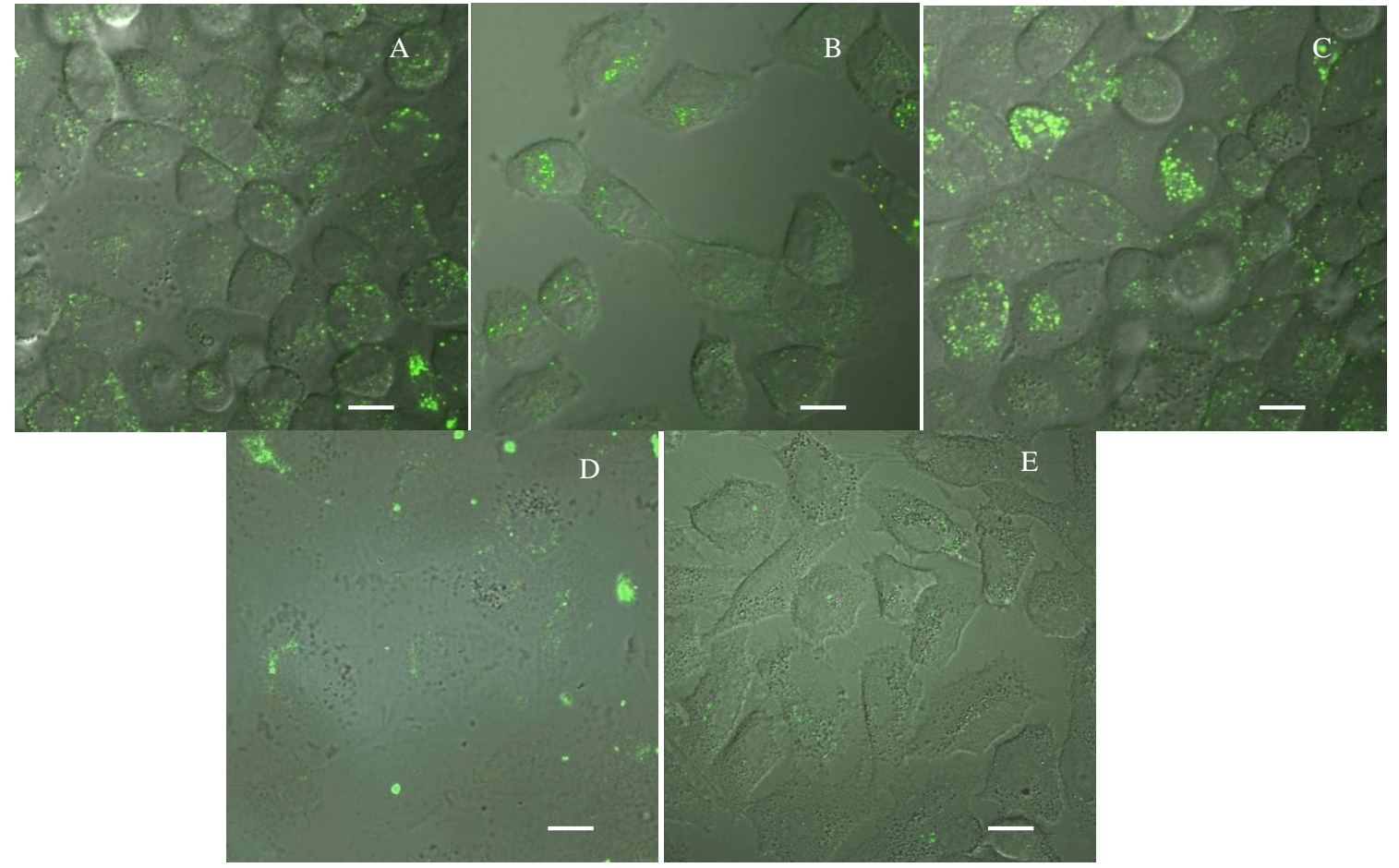

Figure 4: Internalization of the FITC-labeled aptamers into A549 cells after 18 hour incubation in cell culture. A-E: Overlay of the optical images and fluorescence images for A549 cells incubated with R50 (A), R14 (B), R13(C), R9 (D) and Rc (E) Scale bars, $10 \mu \mathrm{m}$.

\subsection{Induced apoptosis of A549 cells by the aptamers}

The cytotoxicity of the aptamers was evaluated using a WST-8 assay with a Cell Counting Kit-8 and Annexin V-FITC/PI apoptosis detection kit after 48 or 24 hours of incubation of A549 cells with the aptamers. When the apoptosis occurred, phosphatidylserine (PS) located normally inside the cell membrane was reversed to extracellular surface and its specific binding to Annexin-V-FITC resulted in the display of green fluorescence on cell membrane. The nuclei in the apoptotic cells would be stained by PI to show red fluorescence. It is interesting that A549 cells incubated with 5 $\mu \mathrm{M}$ aptamers $\mathrm{R} 50$ showed significant apoptosis, as they were stained with both green and red fluorescence clearly (Figure 5A). For R14, a few apoptotic cells were occasionally observed. However, apoptosis of A549 cells was not obvious with R13, R9, and control sequence. Apoptosis induced by R50 was much more obviously than other aptamers.

When $10 \mu \mathrm{M}$ aptamers were used for the cell viability assays, it is found that cell proliferation was inhibited significantly for R50treated cells but not for the cells treated with other aptamers or control sequences (Figure5B). Although R14 exhibit a slight apoptosis in 
confocal experiment, no obvious cytotoxicity was detected in the cell viability assays.

\section{Conclusions}

In this work, EGFR-A549 cells, the NSCLC cells which stably expressing high-level EGFR, were used as the target cells for aptamer selection. EGFR mediates growth signals and its abnormal expression is associated with human cancer. In our previous work using A549 cells as target, aptamers with different sequences (expect R14) which do not have G-rich properties were obtained [21]. This was the first time that several G-rich aptamers were selected from cell-SELEX.

A
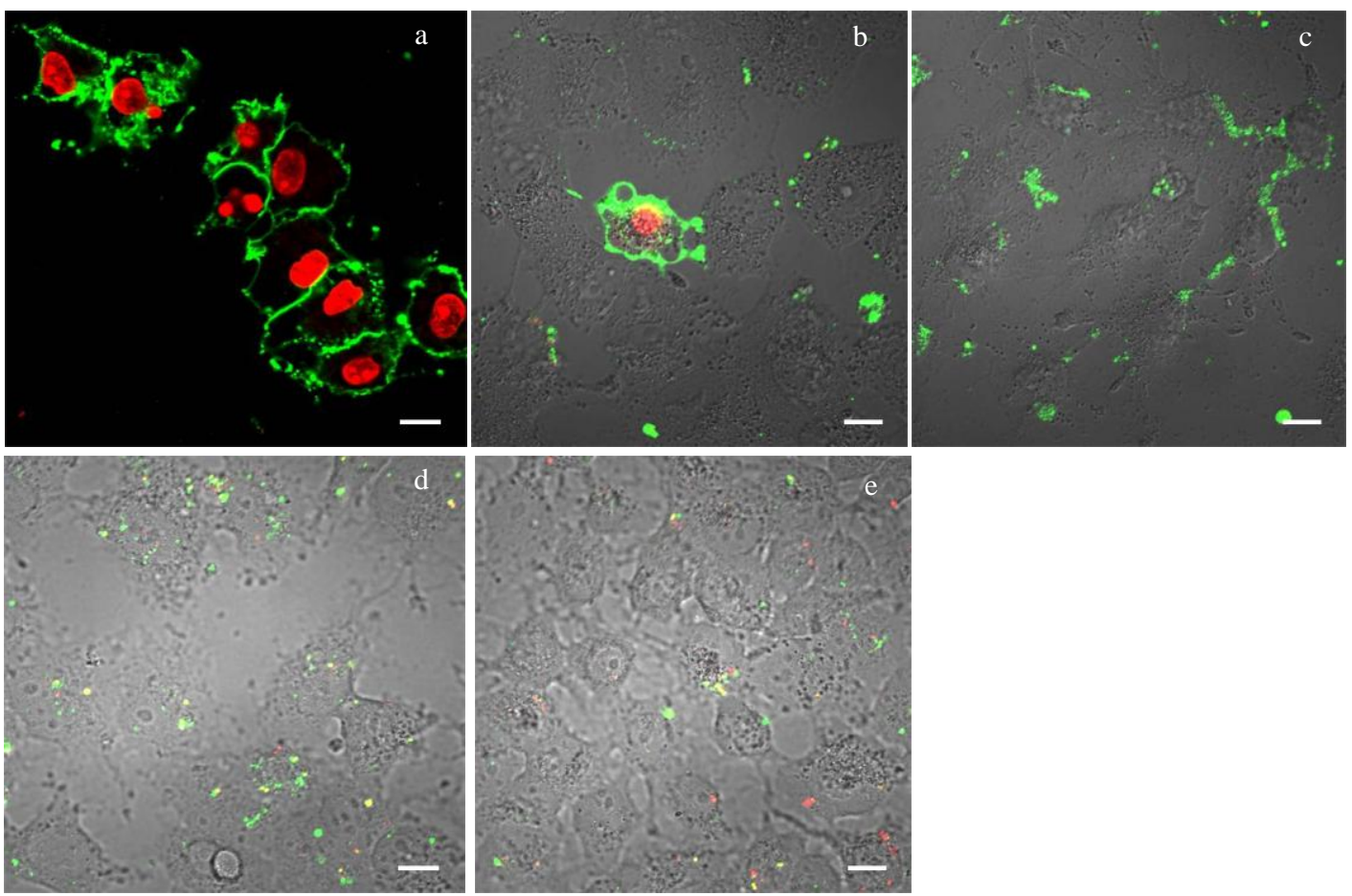

B

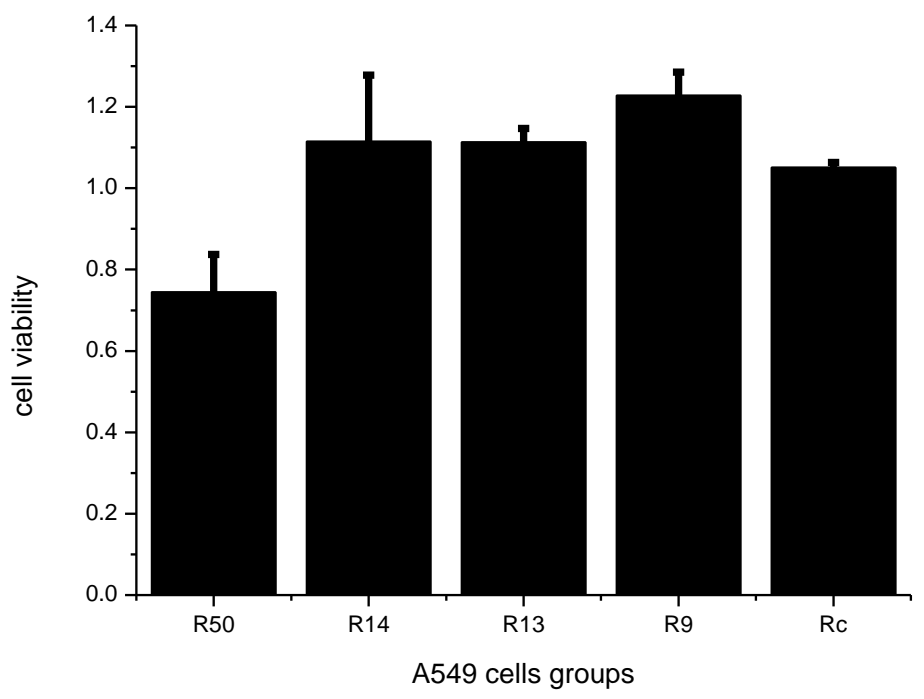

Figure 5: Cytotoxicity evaluation of the selected aptamers. A549 cells were incubated with $5 \mu \mathrm{M}$ aptamers in cell culture for 48 hours for apoptosis evaluation (A) and with $10 \mu \mathrm{M}$ aptamers for 48 hours for viability assay (B). a-e: A549 cells added with R50, R14, R13, R9, and Rc. Scale bars, 10 4 m. 
It has been reported that the nucleolin could bind to some G-rich sequences. Nucleolin, which can undergo nuclear-cytoplasmic shuttling and be present on the cell surface, is involved in cell growth, transcriptional regulation [26-28]. This multifunctional protein could interact with EGFR [29]: binding of nuclear localization sequence of EGFR with the C-terminal 212 amino acids of nucleolin leads to receptor dimerization, activation and tumor growth [30]. The aptamers selected in this work all showed high binding ability to nucleolin, and their binding to EGFRA549 cells with a higher nucleolin expression is stronger that to A549 cells with a lower nucleolin expression. This indicated that the target of selected aptamers might be nucleolin.

Besides high affinity to the NSCLC cells, the selected aptamers had shown their promising properties: all of them could internalize into cells, one of them could inhibit cell proliferation. For the aptamer with both specific cancer-cell affinity and cytotoxicity, it would be developed as drug for cancer therapy [26]. For the aptamers targeting to cancer cells, the ability of their internalization into cells would be beneficial for their application as the carriers for anti-cancer drugs and molecular imaging probes. Previous studies suggested that most aptamers could not be directly taken up by cells due to their negative charge and size, which limited their application in vivo [20, 31-32]. In addition, in this work, we conducted cell-SELEX at $37^{\circ} \mathrm{C}$ in $20 \%$ serum, where cells could be kept in a physiological state. The aptamers we selected could bind to surface of A549 cells and in turn internalize into cells during the selection. Moreover, as the unstable ssDNA in DNA pool might be digested by nuclease in serum during the selection process while stable sequences were left and enriched, we could incubate the cells with the aptamer that added in the normal serum-containing cell medium for a long time (18 hours). The stability is also advantageous for the biomedical application of aptamers.

Among the aptamers selected in our study, R9 had the highest cell affinity while R50 had the lowest. All these aptamers could enter A549 cells. In the cytotoxicity study, R50 showed the highest ability in inducing cell apoptosis and inhibiting cell proliferation. There is no direct relationship between the binding, internalization and cytotoxicity of the aptamers.

In summary, we have selected a series of aptamers for the lung cancer cells. These aptamers showed high affinity to A549 cells. They could internalize into A549 cells without external assistance and had good property of nuclease resistance. They are promising candidates for cancer drug or drug carrier. The study of using the aptamers as targeting reagents for cancer photodynamic therapy and as well as capture probes for cancer diagnosis are under way.

\section{Acknowledgement}

This work was supported by National Basic Research Program of China (2011CB911001), NSFC (No. 21121063) and Chinese Academy of Sciences.

\section{Supplementary information:}

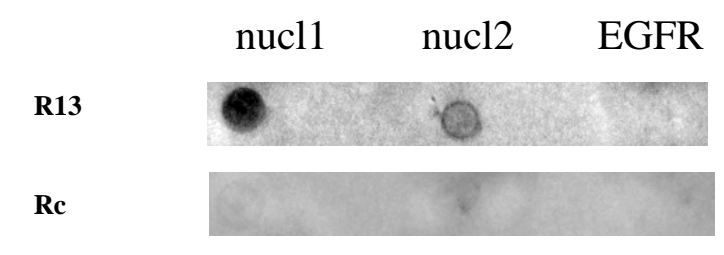

Figure S1: Dot blotting with the biotin-labeled aptamer R13 and the proteins (two nucleolin domains (nuc1: amino acids 323-710, nuc2: amino acids 1-100, and EGFR extracellular domain). The three proteins (nuc1, nuc2 and EGFR extracellular domain from Abcam, England) were diluted to the same concentration according to the instruction and pointed to NC membrane. Each pointed samples of proteins were $0.33 \mu \mathrm{g}$. After dried for 1 hour at room temperature, the samples on the membrane were blocked with $2 \% \mathrm{BSA}$ at $37{ }^{\circ} \mathrm{C}$ for 2 hours and incubated with the $5 \mu \mathrm{M}$ biotin-labeled aptamers at $37^{\circ} \mathrm{C}$ for 1 hour. Then, the membrane was washed with PBS and PBST and incubated with HRP- 
strepavidin at $37^{\circ} \mathrm{C}$ for 1 hour. At last, TMB was used to develop color. Images were obtained by using a digital camera. As indicated by the representative aptamer R13, the aptamer could bind to Nuc1 and Nuc2 but not EGFR; control sequence Rc could bind to none of proteins.

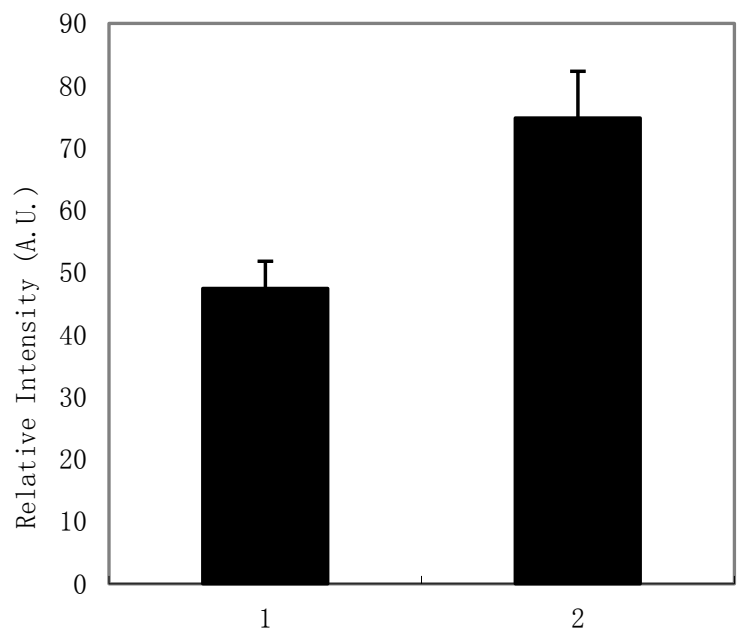

Figure S2: Expression of nucleolin in EGFRA549 and A549 cells. The fluorescence intensities of A549 cells (1) and EGFR-A549 (2) cells were counted. The results indicated the expression of nucleolin was higher in EGFR-A549 than in A549 cells.

\section{References}

[1] Erridge, S. C.; Moller, H.; Price, A.; Brewster,D. International comparisons of survival from lung cancer: pitfalls and warnings. Nat. Clin. Pract.Oncol., 2007, 4(10), 570-577. DOI:10.1038/ncponc0932

[2] Granville, C.A.; Dennis, P.A. An overview of lung cancer genomics and proteomics, Am. J. Respir. Cell Mol. Biol., 2005, 32(3), 169-176 DOI: $10.1165 / \mathrm{rcmb}$. F290.

[3] Praveen, N.; Anshoo, M.; Devinder, K.D. COX-2 as a potential target in chemoprevention of enzo(a)pyrene induced lung carcinogenesis in mice-combined role of curcumin and quercetin, Am. J. Biomed. Sci., 2012, 4(3), 194-203. DOI: $\underline{10.5099 / a j 120300194}$
[4] Buyukcelik, A. B.; Yalcin, B.; Utkan,G. Multidisciplinary management of lung cancer, N. Engl. J. Med., 2004, 350(19), 2008-2010. DOI: $10.1056 /$ NEJMoa040583

[5] Qiu, F.; Liu, H.; Dong, Z.; Feng, Y.; Zhang, X.; Tian, Y. Searching for potential ovarian cancer biomarkers with matrix-assisted laser desorption/ionization time-of-flight mass spectrometry, Am. J. Biomed. Sci., 2009, 1(1), 80-90. DOI: 10.5099/aj090100080

[6] Sung, H.; Cho, J. Biomarkers for the lung cancer diagnosis and their advances in proteomics. BMB Rep, 2008, 41(9), 615-625.

[7] Fang, X.; Tan, W. Aptamers generated from cell-SELEX for molecular medicine: a chemical biology approach, Acc. Chem. Res., 2010, 43(1), 48-57. DOI: 10.1021/ar900101s

[8] Shangguan, D.; Li, Y.; Tang, Z.; Cao, Z.; Chen, H.; Mallikaratchy, P.; Sefah, K.; Yang, C.; Tan, W. Aptamers evolved from live cells as effective molecular probes for cancer study, Proc. Natl. Acad. Sci. U.S.A., 2006,103(32), 11838-11843. DOI: 10.1073/pnas.0602615103

[9] Shangguan, D.; Cao, Z.; Li, Y.; Tan, W. Aptamers evolved from cultured cancer cells reveal molecular differences of cancer cells in patient samples, Clin. Chem., 2007, 53(6), 1153-1155. 10.1373/clinchem.2006.083246

[10]Jayasena, S. D. Aptamers: an emerging class of molecules that rival antibodies in diagnostics, Clin. Chem., 1999, 45(9), 16281650.

[11] Yang, C.; Jockusch, S.; Vicens, M.; Turro, N.J.; Tan, W. Light-switching excimer probes for rapid protein monitoring in complex biological fluids, Proc. Natl. Acad Sci. U.S.A., 2005, 102(48), 17278-17283. DOI: 10.1073/pnas.0508821102

[12] Breaker, R. R. Natural and engineered nucleic acids as tools to explore biology, Nature, 2004, 432(7019), 838-845. DOI:10.1038/nature03195

[13] Chen, H.; Medley, C. D.; Sefah, K.; Shangguan, D.; Tang, Z.; Meng, L.; Smith, J. E.; Tan, W. Molecular recognition of smallcell lung cancer cells using aptamers, Chem. Med. Chem., 2008, 3(6), 991-1001. DOI: 
$10.1002 / \mathrm{cmdc} .200800030$

[14] Mallikaratchy ,P.; Tang, Z.; Sefah,K.; Meng, L.; Shangguan, D.; Tan ,W. Aptamer directly evolved from live cells recognizes membrane bound immunoglobin heavy mu chain in Burkitt's lymphoma cells, Mol. Cell Proteomics, 2007, 6(12), 2230-2238. DOI: 10.1074/mcp.M700026-MCP200

[15] Shangguan, D.; Cao Z.; Meng, L.; Mallikaratchy ,P.; Sefah,K.; Wang, H.; Li, Y.; Tan ,W. Cell-specific aptamer probes for membrane protein elucidation in cancer cells, J. Proteome Res., 2008, 7(5), 2133-2139. DOI: $10.1021 / \mathrm{pr} 700894 \mathrm{~d}$

[16]Lupold, S.E.; Hicke, B.J.; Lin, Y.; Coffey, D.S. Identification and characterization of nuclease-stabilized RNA molecules that bind human prostate cancer cells via the prostatespecific membrane antigen, Cancer Res., 2002, 62(14), 4029-4033.

[17]Ferreira, C.S.; Cheung, M.C.; Missailidis, S.; Bisland, S.; Gariepy, J. Phototoxic aptamers selectively enter and kill epithelial cancer cells, Nucleic Acids Res., 2009, 37(3), 866876. DOI: $10.1093 / \mathrm{nar} / \mathrm{gkn} 967$

[18] McNamara, J.O. II,; Andrechek, E.R.; Wang,Y.; Viles,K.D.; Rempel,R.E.; Gilboa,E.; Sullenger,B.A.; Giangrande,P.H. Cell type-specific delivery of siRNAs with aptamer-siRNA chimeras, Nat. Biotechnol., 2006, 24(8), 1005-1015. DOI:10.1038/nbt1223

[19]Thiel, K.W.; Hernandez, L.I.; Dassie, J.P.; Thiel, W.H.; Liu, X.; Stockdale, K.R.; Rothman, A.M.; Hernandez, F.J.; McNamara, J.O. II.; Giangrande, P.H. Delivery of chemosensitizing siRNAs to HER2+-breast cancer cells using RNA aptamers, Nucleic Acids Res., 2012, 40(13), 6319-6337. DOI: $\underline{10.1093 / \mathrm{nar} / \mathrm{gks} 294}$

[20]Xiao, Z.; Shangguan, D.; Cao, Z.; Fang, X.; Tan, W. Cell-specific internalization study of an aptamer from whole cell selection, Chem. Eur. J., 2008, 14(6), 1769-1775. DOI: 10.1002/chem.200701330

[21]Zhao, Z.; Xu, L.; Shi, X.; Tan, W.; Fang, X.; Shangguan, D. Recognition of subtype nonsmall cell lung cancer by DNA aptamers selected from living cells, Analyst, 2009,
134(9), $\quad 1808-1814$.

DOI: 10.1039/B904476K

[22]Zhang, Z.; Zhao, Z.; Xu, L.; Wu, X.; Zhu,H.; Tan, W.; Fang, X. Hepatitis C virus core protein detection by DNA aptamer, SCIENTIA SINICA Chimica, 2011, 41(8), 1312-1318. DOI: 10.1360/032011-198

[23] Sgambato, A.; Casaluce, F.; Maione, P.; Rossi, A.; Rossi, E.; Napolitano, A.; Palazzolo, G.; Bareschino, M.; Schettino,C.; Sacco, P.C.; Ciardiello, F.; Gridelli, C. The role of EGFR tyrosine kinase inhibitors in the first-line treatment of advanced non small cell lung cancer patients harboring EGFR mutation, Curr. Med. Chem., 2012, 19(20), 3337-3352. $\underline{10.2174 / 092986712801215973}$

[24] Mongelard, F.; Bouvet, P. AS-1411, a guanosine-rich oligonucleotide aptamer targeting nucleolin for the potential treatment of cancer, including acute myeloid leukemia. Curr. Opin. Mol. Ther, 2010, 12(1):107-114.

[25]Bayrac, A.T.; Sefah, K.; Parekh, P.; Bayrac, C.; Gulbakan, B.; Oktem, H.A.; Tan, W. In vitro Selection of DNA Aptamers to Glioblastoma Multiforme, ACS Chem. Neurosci., 2011, 2(3), 175-181. DOI: $\underline{10.1021 / \mathrm{cn} 100114 \mathrm{k}}$

[26] Ugrinova, I.;Monier, K.; Ivaldi, C.; Thiry, M.; Storck, S.; Mongelard, F.; Bouvet, P. Inactivation of nucleolin leads to nucleolar disruption, cell cycle arrest and defects in centrosome duplication, BMC.Mol.Biol., 2007;8:66. DOI: 10.1186/1471-2199-8-66

[27] Stepanova,V.;Lebedeva,T.; Kuo, A.; Yarovoi, S.; Tkachuk, S.; Zaitsev, S.; Bdeir, K.; Dumler, I.; Marks, M.S.; Parfyonova, Y.; Tkachuk, V.A.; Hiqazi, A.A.; Cines, D.B. Nuclear translocation of urokinase-type plasminogen activator, Blood, 2008, 112(1), 100-110. DOI:10.1182/blood-2007-07104455

[28]Joo, E.J.; Yang, H.; Park, Y.; Park, N.Y.; Toida, T.; Linhardt, R.J.; Kim, Y.S. Induction of nucleolin translocation by Acharan sulfate in A549 Human Lung Adenocarcinoma, J. Cell. Biochem., 2010, 110, 1272-1278. DOI: 10.1002/jcb.22643

[29]DiSegni, A.; Farin, K.; Pinkas-Kramarski, R. 
Identification of nucleolin as new ErbB receptors-interacting protein. PLoSOne, 2008, 3(6):e2310. DOI: 10.1371/journal.pone.0002310

[30]Farin, K.; Schokoroy, S.; Haklai, R. Oncogenic Synergism between ErbB1, Nucleolin, and Mutant Ras. Cancer Res., 2011, 71, 2140-2151. DOI: 10.1158/00085472.CAN-10-2887

[31]Chu,T.C.; Marks, J.W. 3rd; Lavery, L.A.; Faulkner,S.;
Rosenblum,M.G.;
Ellington,A.D.; Levy,M. Aptamer:toxin conjugates that specifically target prostate tumor cells, Cancer Res., 2006, 66(12), 59895992. DOI: 10.1158/0008-5472.CAN-102887

[32] Patil,S.D.; Rhodes, D.G.; Burgess, D.J. DNA-based therapeutics and DNA delivery systems: a comprehensive review, AAPS $J$. 2005, 7(1), E61-E77. DOI: 